\title{
Magnetic resonance imaging-derived radiomic signature predicts locoregional failure after organ preservation therapy in patients with hypopharyngeal squamous cell carcinoma
}

\author{
Che-Yu Hsu ${ }^{\text {a,b,c,1 }}$, Shih-Min Lin ${ }^{\text {e,1 }}$, Ngan Ming Tsang ${ }^{\text {e,h }}$, Yu-Hsiang Juan ${ }^{\text {f }}$, Chun-Wei Wang ${ }^{\text {a,b,c }}$, \\ Wei-Chung Wang ${ }^{g}$, Sung-Hsin Kuo ${ }^{\mathrm{a}, \mathrm{b}, \mathrm{c}, \mathrm{d}, *}$ \\ ${ }^{a}$ Division of Radiation Oncology, Department of Oncology, National Taiwan University Hospital, Taipei, Taiwan \\ ${ }^{\mathrm{b}}$ Department of Radiation Oncology, National Taiwan University Cancer Center, National Taiwan University College of Medicine, Taipei, Taiwan \\ ${ }^{\mathrm{c}}$ Cancer Research Center, National Taiwan University College of Medicine, Taipei, Taiwan \\ ${ }^{\mathrm{d}}$ Graduate Institute of Oncology, National Taiwan University College of Medicine, Taipei, Taiwan

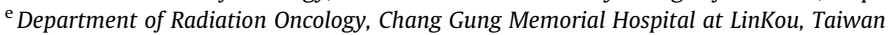 \\ ${ }_{\mathrm{f}}^{\mathrm{f}}$ Department of Medical Imaging and Intervention, Chang Gung Memorial Hospital at Taoyuan, Taiwan \\ ${ }^{\mathrm{g}}$ Department of Mathematics, National Taiwan University, Taipei, Taiwan \\ ${ }^{\mathrm{h}}$ School of Traditional Chinese Medicine, Chang Gung University, Taoyuan, Taiwan
}

\section{A R T I C L E I N F O}

\section{Article history:}

Received 12 May 2020

Revised 16 August 2020

Accepted 24 August 2020

Available online 31 August 2020

\section{Keywords:}

Hypopharyngeal squamous cell carcinoma

Organ preservation treatment

Radiomics

Loco-regional failure

Survival

\begin{abstract}
A B S T R A C T
Background and purpose: To develop and validate a magnetic resonance imaging (MRI)-derived radiomic signature (RS) for the prediction of 1-year locoregional failure (LRF) in patients with hypopharyngeal squamous cell carcinoma (HPSCC) who received organ preservation therapy (OPT)

Material and methods: A total of 800 MRI-based features of pretreatment tumors were obtained from 116 patients with HPSCC who received OPT from two independent cohorts. The least absolute shrinkage and selection operator regression model were used to select the features used to develop the RS. Harrell's Cindex and corrected C-index were used to evaluate the discriminative ability of RS. The Youden index was used to select the optimal cut-point for risk category.

Results: The RS yielded 1000 times bootstrapping corrected C-index of 0.8036 and 0.78235 in the experimental $(n=82)$ and validation cohorts $(n=34)$, respectively. With respect to the subgroup of patients with stage III/IV and cT4 disease, the RS also showed good predictive performance with corrected Cindices of 0.760 and 0.754 , respectively. The dichotomized risk category using an RS of 0.0326 as the cut-off value yielded a 1-year LRF predictive accuracy of 79.27\%, 79.41\%, 76.74\%, and 71.15\% in the experimental, validation, stage III/IV, and cT4a cohorts, respectively. The low-risk group was associated with a significantly better progression-free laryngectomy-free and overall survival outcome in two independent institutions, stage III/IV, and cT4a cohorts.

Conclusion: The RS-based model provides a novel and convenient approach for the prediction of the 1year LRF and survival outcome in patients with HPSCC who received OPT.

(C) 2020 The Author(s). Published by Elsevier B.V. on behalf of European Society for Radiotherapy and

Oncology. This is an open access article under the CC BY-NC-ND license (http://creativecommons.org/
\end{abstract} licenses/by-nc-nd/4.0/).

\section{Introduction}

Hypopharyngeal squamous cell carcinomas (HPSCC) account for approximately $3 \%$ to $5 \%$ of all head and neck cancers (HNCs) $[1,2]$. Patients with HPSCC often present with a locally advanced disease

\footnotetext{
* Corresponding author at: Division of Radiation Oncology, Department of Oncology, National Taiwan University Hospital, No. 7, Chung-Shan South Rd, Taipei 100, Taiwan.

E-mail address: shkuo101@ntu.edu.tw (S.-H. Kuo).

1 Che-Yu Hsu and Shih-Min Lin contribute equally to this work.
}

and have a poor prognosis despite the diagnostic and therapeutic improvements in this disease area [2-5]. During the past decade, in addition to the mainstay treatment of total laryngectomy (TL) for locally advanced HPSCC, the increased application of organ preservation treatment (OPT), including induction chemotherapy (IC) followed by definitive radiotherapy (RT), or primary concurrent chemoradiotherapy (CCRT) has resulted in an increase in the 5 -year overall survival (OS) rate from $38 \%$ to $51.9 \%[2,6]$. Because of comparable outcomes with TL and the benefit of preservation of the larynx, the OPT is considered as an alternative therapeutic choice for advanced HPSCC patients [2,6]. However, for persistent 
hypopharyngeal tumors after OPT, salvage surgery may result in complications such as wound infection, wound dehiscence, and pharyngocutaneous fistula $[7,8]$. Therefore, development of a model for the accurate prediction of the tumor response and tumor control after OPT, in patients with locally advanced HPSCC, is warranted.

The clinical tumor-node-metastasis (TNM) stage system developed by the American Joint Committee on Cancer (AJCC) is currently used to classify HPSCC into early- and advanced-stage cancers using measures of tumor extent $[9,10]$. Previous studies have reported that a higher clinical $\mathrm{T}$ (cT) and lymph node $(\mathrm{cN})$ parameters are closely associated with poor disease control in HPSCC $[9,10]$. A series of studies have demonstrated that the tumor volume (TV) staging approach is superior to the TNM staging system for the prediction of local disease control in patients with HNC [11-14]. For example, a TV of $>30$ or $35 \mathrm{~cm}^{3}$ is a prognostic factor for poor disease outcome in patients with HPSCC $[13,14]$.

Radiomics, an emerging field of quantitative image analysis, is recognized for noninvasively obtaining quantitative, threedimensional features of neoplastic tissue from serial images and thus providing informative radiographic-imaging phenotypes of entire tumors, including TV, tumor shape, and intratumoral heterogeneity [15-18]. Radiomic signature (RS), the combined analysis of a panel of radiomic features, is a promising approach that may facilitate the individualized prediction of the prognosis in several kinds of cancers [17-19]. Computed tomography (CT)-based radio- mics analysis has been found to be associated with prognosis in patients with HNC $[15,17,20]$. However, there is no optimal approach that combines the multiple image features of magnetic resonance imaging (MRI) and RS for the prediction of persistent or early loco-regional recurrent diseases, post OPT, in patients with HPSCC.

We hypothesized that 1-year loco-regional failure (LRF) events, including persistent or recurrent primary tumors and regional lymphadenopathies, may reflect failure of OPT in patients with locally advanced HPSCC who received CCRT or RT as a first-line treatment. Therefore, we sought to develop an MRI based RS score to predict the 1-year LRF events in the experimental cohort of HPSCC patients who received a cisplatin-based CCRT as the primary treatment, and validate the RS classifier in the validation cohort (cisplatin-based CCRT as the primary treatment). Furthermore, the use of the high-risk and low-risk classification system based on the RS score may help us select suitable patients for OPT without compromising the disease prognosis.

\section{Materials and methods}

\subsection{Study population}

Ninety and twenty-six histologically confirmed HPSCC patients, diagnosed between January 2003 and December 2010 at the Institution 1 (Chang-Gung Memorial Hospital) and between January
A

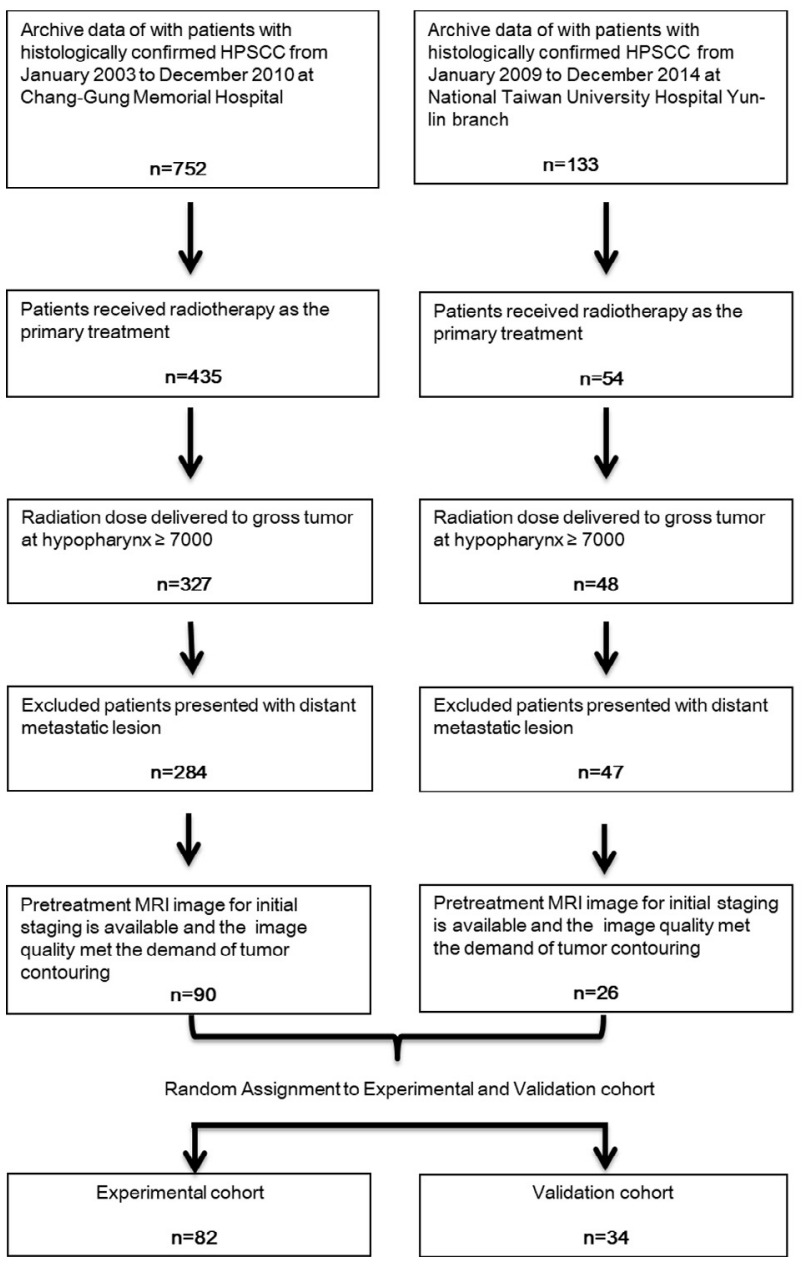

B
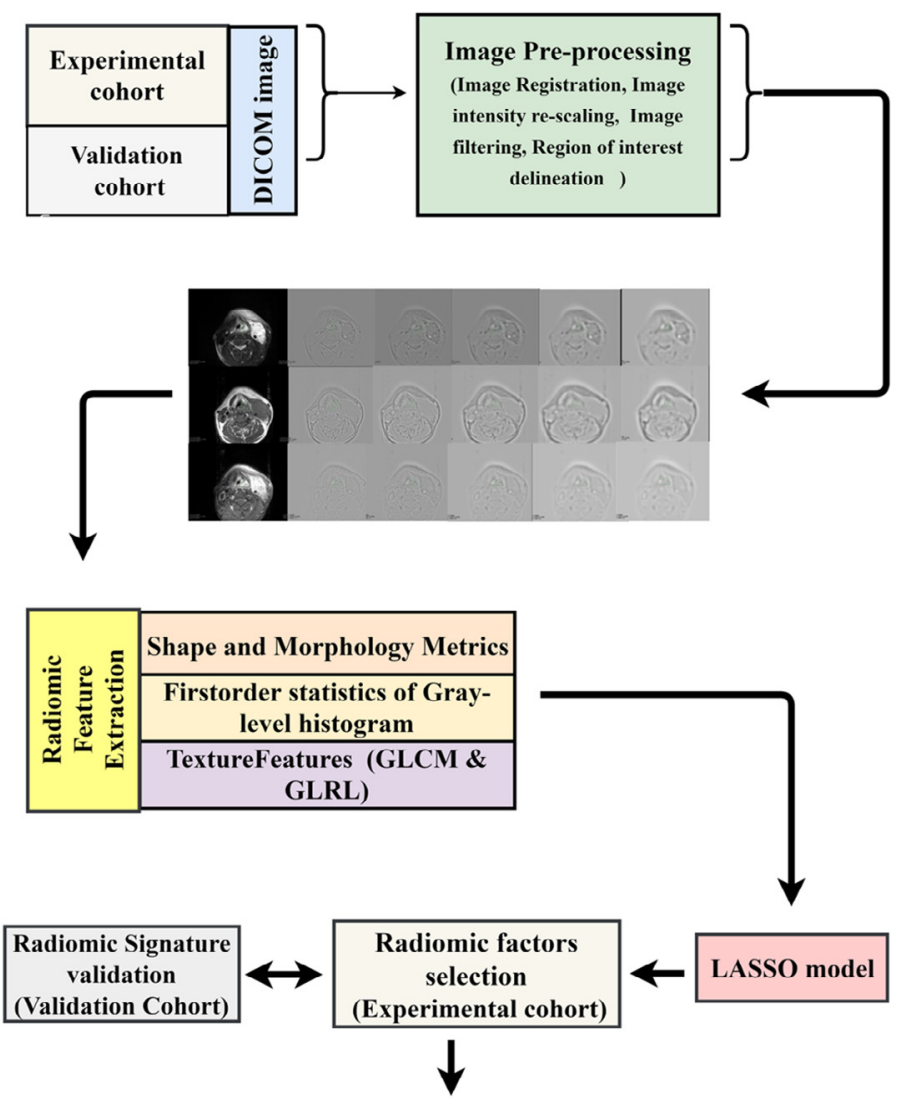

Radiomic Signature

(prediction of loco-regional failure in Hypopharyngeal cancer)

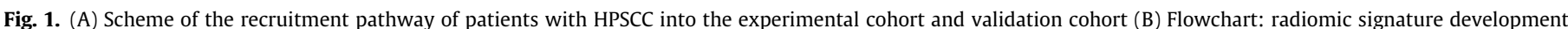

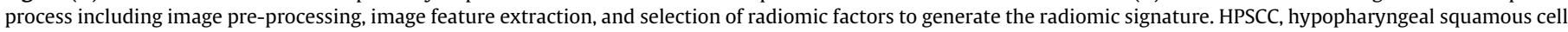
carcinoma; GLCM, gray-level co-occurrence matrix; GLRL, gray-level run-length; LASSO, the least absolute shrinkage and selection operator. 
2009 and December 2014 at the Institution 2 (Yun-Lin branch of the National Taiwan University Hospital), who received a cisplatin-based CCRT as the first-line treatment for OPT were respectively enrolled in the present study. All patients underwent a pretreatment MRI scanning for initial staging, and none of the patients who underwent tracheotomy were included in our cohort. The surviving patients were followed-up for a minimum of 2 years. Patients who did not complete the whole course of RT (radiation dose to gross tumors $\geq 70 \mathrm{~Gy}$ ), those who received surgery as primary treatment, or those who initially presented distant metastatic lesions were excluded from the study. From the entire cohort, 82 and 34 patients were randomly assigned to the experimental and validation cohort, respectively. The recruitment pathway of all the patients is depicted in Fig. 1A. The baseline demographical data of age, sex, histological grade, and TNM stage (according to the 7th edition of the AJCC TNM staging system) of the experimental and validation cohorts were obtained from the medical records, and the demographic details are listed in Table 1. The Research Ethical Committee of the Chang-Gung Memorial Hospital and the National Taiwan University Hospital approved the retrospective study protocol.

\subsection{MRI imaging protocols}

All patients were examined with MRI before and after gadolinium-DTPA injection. A head-and-neck synergic coil covering the entire neck, from the skull base to the thoracic inlet, was used for the MRI examinations. Pre-contrast T2- and T1-weighted turbo spin-echo (TSE) images were obtained in the axial plane, and post-contrast T1-weighted TSE images with fat saturation were obtained in the axial, coronal, and sagittal planes. The MRI was performed with a 1.5-T unit (Intera, Philips Medical Systems; $\mathrm{n}=11$ ) or 1.5 -T MRI system (Signa HDxt; GE Healthcare; $\mathrm{n}=26$ ) or a 3-T unit (Magnetom Trio, Siemens Healthcare, Erlangen, Germany, $\mathrm{n}=79$ ). Supplementary materials and methods S1 show the protocol associated with the imaging parameters used for the MRI sequences.

Table 1

Baseline patient characteristics in the experimental and validation cohorts.

\begin{tabular}{|c|c|c|c|}
\hline & $\begin{array}{l}\text { Experimental } \\
\text { Cohort }\end{array}$ & $\begin{array}{l}\text { Validation } \\
\text { Cohort }\end{array}$ & $\begin{array}{l}P \\
\text { value }\end{array}$ \\
\hline \multicolumn{4}{|l|}{ Age, n (\%) } \\
\hline$>60$ years & $15(18.3)$ & $6(17.6)$ & 0.934 \\
\hline$\leq 60$ years & $67(81.7)$ & $28(82.4)$ & \\
\hline \multicolumn{4}{|l|}{ Sex, n (\%) } \\
\hline Male & $78(95.1)$ & $33(97.1)$ & 0.640 \\
\hline Female & $4(4.9)$ & $1(2.9)$ & \\
\hline \multicolumn{4}{|l|}{ Clinical T stage, n (\%) } \\
\hline T1-T3 & $30(36.6)$ & $15(44.1)$ & 0.449 \\
\hline $\mathrm{T} 4$ & $52(63.4)$ & $19(55.9)$ & \\
\hline \multicolumn{4}{|l|}{ Clinical N stage, n (\%) } \\
\hline NO & $13(15.9)$ & $9(26.5)$ & 0.184 \\
\hline N1-N3 & $69(84.1)$ & $25(73.5)$ & \\
\hline \multicolumn{4}{|l|}{ Clinical TNM stage, n (\%) } \\
\hline Stage I-III & 13 (15.9) & $9(26.5)$ & 0.184 \\
\hline Stage IV & $69(84.1)$ & $25(73.5)$ & \\
\hline \multicolumn{4}{|l|}{ Histologic grade, $\mathrm{n}(\%)$} \\
\hline Grade $1-2$ & 77 (93.9) & $29(85.3)$ & 0.133 \\
\hline Grade 3 & $5(6.1)$ & $5(14.7)$ & \\
\hline Tumor volume, mean $\pm \mathrm{SD}, \mathrm{mm}^{3}$ & $36.67 \pm 30.66$ & $34.7 \pm 39.2$ & 0.778 \\
\hline $\begin{array}{l}\text { Follow-up time, mean } \pm \mathrm{SD}, \mathrm{mm}^{3} \text {, } \\
\text { month }\end{array}$ & $39.34 \pm 34.65$ & $49.45 \pm 36.84$ & 0.163 \\
\hline \multicolumn{4}{|l|}{ Institution, $n(\%)$} \\
\hline Institution 1 & $65(79.3)$ & $25(73.5)$ & 0.500 \\
\hline Institution 2 & $17(20.7)$ & $9(26.5)$ & \\
\hline
\end{tabular}

Abbreviations: n, number; T, tumor; N, lymph node, SD, standard deviation.
2.3. Image preprocessing and quantitative image analysis workflow used to create the radiomic signature

The following experiments, including image preprocessing, quantitative image analysis, and workflow to create the RS from the experimental and validation cohort are illustrated in Fig. 1B. All images were reviewed and processed using the 3D Slicer software (3D Slicer, Version 4.5.0-1, http://www.slicer.org) [21]. First, 6-Degrees-of-Freedom (6-DOF) Rigid-Body registration was applied to the T2-weighted (T2), non-contrast T1-weighted (T1n), and contrast enhanced T1-weighted (T1c) MRI images. Based on the MRI images, a region of interest (ROI) was delineated around the entire visible tumor by a radiation oncologist (C.Y.H., with 7 years of experience in HNC contouring). After the ROIs were defined, a second radiation oncologist (S.M.L. with 7 years of experience in HNC contouring) re-evaluated 30 randomly chosen MRI images for the assessment of the inter-observer variations. Both the observers were blinded to the clinical data and the pathology reports of the patients before and during the contouring process. Image gray-level normalization, known to minimize arbitrary MR signal intensities within the standardized intensity ranges, was performed by rescaling the histogram data into 256 Gy levels (Supplementary materials and methods S2).

Next, we applied a Laplacian of Gaussian filter (LoG) to the intensity-normalized $\mathrm{T} 2, \mathrm{~T} 1 \mathrm{n}$ and T1c MRI images with filter parameters between 1.0 and $3.0(1.0,1.5,2.0,2.5,3.0)$ to improve suppression of image noise and enrich the features of the diverse spatial scales (Supplementary materials and methods S2) [21,22]. The total radiomic features, extracted from the intensitynormalized T2, T1n, and T1c MRI images, with or without LoG image filtration (18 sets of MRI images, totally), consisted of (i) $4 \mathrm{vol}$ and 4 shape features (ii) 270 image features of first-order statistics of gray-level histograms (iii) 324 texture features derived from gray-level co-occurrence matrix (GLCM) analysis (iv) 198 texture features derived from the gray-level run-length (GLRL) matrix. We utilized the heterogeneity computer-aid diagnosis (CAD) module of 3D slicer as the feature extraction tool [18,21,23-26].

The least absolute shrinkage and selection operator (LASSO) is an L1-regularized regression method and allows a panel of selected features to be combined into an RS $[27,28]$. In the experimental cohort, we input the radiomic features extracted from the MRI images and used the LASSO binary logistic regression tool of the lasso and elastic-net regularized generalized linear models (GLMNET) package in the software R (version 3.3.1; http://www. Rproject.org) to build the RS to predict the 1-year LRF events. We employed a 10-fold cross-validation method, using the minimum criteria with the measure type "Class", to tune the parameter $\lambda$ in the LASSO model. The chosen parameter $\lambda$ could help select valuable image features with non-zero coefficients.

\subsection{Assessment of the association between the clinicopathological} features and the radiomic signature with 1-year loco-regional control

Univariate analysis was performed to evaluate the association between the clinicopathological factors and 1-year LRF events in the experimental and validation cohorts. Harrell's C-index and the corrected $\mathrm{C}$-index, derived from the bootstrapping validation with 1000 resamples to reduce potential overfitting, were calculated by using the "Hmisc" package in the R software in order to evaluate discriminative ability of RS for the 1-year LRF.

\subsection{Follow-up and statistical analysis of the clinical outcomes}

After completion of the OPT, all patients were followed up every 1 to 2 months in the first 2 years, and every 3 to 4 months thereafter. During each follow-up, a physical examination and 
laryngoscopy were performed, and a CT or MRI of the head and neck region was performed every 4 to 6 months in the first $1-$ 2 years. The median follow-up time for those still alive at the last follow-up was 91 months (range: 27.67-137.03 months). The diagnosis of LRF was based on the laryngoscopy results, CT or MRI of the head and neck region, or both (if needed, tissue proof or fineneedle aspiration was performed). Distant failure (DF) was diagnosed based on the physical examination, metastases-related symptoms, and imaging modalities including chest radiography, abdominal sonography, or CT/MRI/whole body bone/positron emission tomography (PET). Progression-free survival (PFS) was measured from the first day of treatment to the date of disease progression including LRF, DF, or death. Laryngectomy-free survival (LFS) was measured from the date of the diagnosis of HPSCC until the date of TL or death. OS was measured from the date of the diagnosis of HPSCC to death. Survival analysis was calculated using the Kaplan-Meier method. A $P$ value of $<0.05$ was considered statistically significant.

\section{Results}

\subsection{Demographic findings of all patients}

The demographic findings of the experimental and validation cohorts are listed in Table 1. The median age of the entire cohort was 51 (range: 34-78) years, and most patients (95.7\%) were men. There were no significant differences between the two cohorts in the distribution of elderly patients $(P=.934)$, gender $(P=.640), \mathrm{cT}$ category $(P=.449), \mathrm{cN}$ category $(P=.184)$, histological grading $(P=.133)$, and primary TV $(P=.778)$.

\subsection{Feature selection and radiomic signature building}

We developed the RS, using the LASSO method, from 800 radiomic features obtained from 82 patients in the experimental cohort. A lambda $(\lambda)$ value of 0.067490 gave a regularized model that allowed the mean cross-validated error to be within one standard error of the minimum value, and aided in narrowing down the 800 image features to 8 potential radiomic predictors. By means of the aforementioned 8 potential radiomic predictors, we developed the new RS to assess the association with the 1-year LRF in the experimental cohort. The radiomic features are listed in Supplementary Results S1, and the formula for RS is listed below:

$$
\begin{aligned}
R S= & 0.619630267+(-0.336355932) \\
& \times \text { Surface.Volume.Ratio }+(0.12324892) \times \text { Compactness. } 1 \\
& +(0.007160570) \times \text { Kurtosis }_{T} 2_{2}+(0.038133581) \\
& \times \text { Maximum } .
\end{aligned}
$$

IntensityT1n1 + $(-0.016441710) \times$ SkewnessT1n1

$$
\begin{aligned}
& +(0.001521672) \times \text { Kurtosis }_{T} 1 n_{1}+(-0.019342842) \\
& \times \text { Kurtosis }_{T} 1 c_{0}+(-0.005920437) \times \text { LGLRE }_{T} 1 c_{2.5}
\end{aligned}
$$

With regards to the inter-observer agreement assessment between 2 readers for the features of the RS, we found that the intraclass correlation coefficients ranged from 0.787 to 0.9259 (Supplementary Results S2). As the coefficients were $>0.7$, this result indicated a good agreement of the assessment between the 2 readers.

\subsection{Association of the clinicopathological features and radiomic signature with 1-year loco-regional failure events}

In the experimental cohort, in addition to the cT, TV and TNM staging, the RS was significantly higher in patients with a 1-year LRF $(P<.001)$. Among these significant prognostic factors in the experimental cohort, only TV and RS were found to be significant in the validation cohort, whereas RS was still significantly higher in patients with a 1 -year $\operatorname{LRF}(P=.006)$ (Table 2 and Fig. $2 A$ ).

The RS yielded AUC of ROC curves of 0.804 (95\% confidence interval [CI], 0.708-0.899) and 0.784 (95\% CI, 0.598-0.971) in the experimental and validation cohorts, respectively (Fig. 2B). On bootstrapping validation (1-year LRF), the corrected C-indices in the experimental and validation cohorts were 0.8036 and 0.78235 , respectively (Supplementary Results S3). As for the subgroup of patients with stage III /IV and cT4 disease, which were regarded as technically resectable but locally advanced diseases, the RS also showed good predictive performance with bootstrap corrected C-indices of 0.7609 and 0.7545 , respectively (Supple-

\begin{tabular}{|c|c|c|c|c|c|c|}
\hline & \multicolumn{3}{|c|}{ Experimental cohort } & \multicolumn{3}{|l|}{ Validation cohort } \\
\hline & 1-year LR control & 1-year LR failure & $P$ value & 1-year LR control & 1-year LR failure & $P$ value \\
\hline \multicolumn{7}{|l|}{ Age, year } \\
\hline Mean \pm SD & $55.04 \pm 9.15$ & $51.00 \pm 9.56$ & 0.054 & $51.77 \pm 8.62$ & $51.58 \pm 9.25$ & 0.953 \\
\hline \multicolumn{7}{|l|}{ Smoking, n (\%) } \\
\hline No & $13(31.7)$ & $11(26.8)$ & 0.809 & $8(50)$ & $5(50)$ & 1.000 \\
\hline Yes & $28(68.3)$ & $30(73.2)$ & & $8(50)$ & $5(50)$ & \\
\hline \multicolumn{7}{|l|}{ cT, n (\%) } \\
\hline T1-T3 & $21(51.2)$ & $9(22.0)$ & 0.011 & $11(68.8)$ & $4(40)$ & 0.228 \\
\hline $\mathrm{T} 4$ & $20(48.8)$ & $32(78.0)$ & & $5(31.2)$ & $6(60)$ & \\
\hline \multicolumn{7}{|l|}{$\mathrm{cN}, \mathrm{n}(\%)$} \\
\hline NO-N1 & $9(22.0)$ & $4(9.8)$ & 0.226 & $13(81.2)$ & $3(30)$ & 0.015 \\
\hline N2-N3 & $32(78.0)$ & $37(90.2)$ & & $3(18.8)$ & $7(70)$ & \\
\hline \multicolumn{7}{|l|}{ TNM, n (\%) } \\
\hline Stage I-III & $9(22.0)$ & $4(9.8)$ & 0.226 & $9(56.3)$ & $3(30)$ & 0.226 \\
\hline Stage IV & $32(78.0)$ & $37(90.2)$ & & $7(43.8)$ & $7(70)$ & \\
\hline \multicolumn{7}{|l|}{$\mathrm{HG}, \mathrm{n}(\%)$} \\
\hline Grade $1-2$ & 38 (92.7) & $39(95.1)$ & 1.000 & $15(93.8)$ & $10(100)$ & 1.00 \\
\hline Grade 3 & $3(7.3)$ & $2(4.9)$ & & $1(6.2)$ & $0(0)$ & \\
\hline \multirow{2}{*}{$\begin{array}{l}\mathrm{TV}, m^{3} \text { mean } \pm \mathrm{SD}, \mathrm{RS} \\
\text { mean } \pm \mathrm{SD}\end{array}$} & $25.59 \pm 24.68$ & $47.73 \pm 32.30$ & 0.001 & $22.93 \pm 29.47$ & $56.40 \pm 46.56$ & 0.015 \\
\hline & $-0.36 \pm 0.66$ & $0.34 \pm 0.54$ & $<0.001$ & $-0.46 \pm 0.69$ & $0.36 \pm 0.94$ & 0.006 \\
\hline
\end{tabular}
mentary Results S3). We further performed c-statistics to analyze the discriminative performance of the RS in predicting 2-year LRF and found that the RS showed good predictive performance, with

Table 2

The distribution of prognostic factors in 1-year LR control and LR failure subgroups in experimental and validation cohorts.

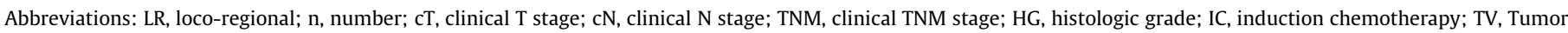
volume; SD, standard deviation; Q1, first quartile; Q3, third quartile. 

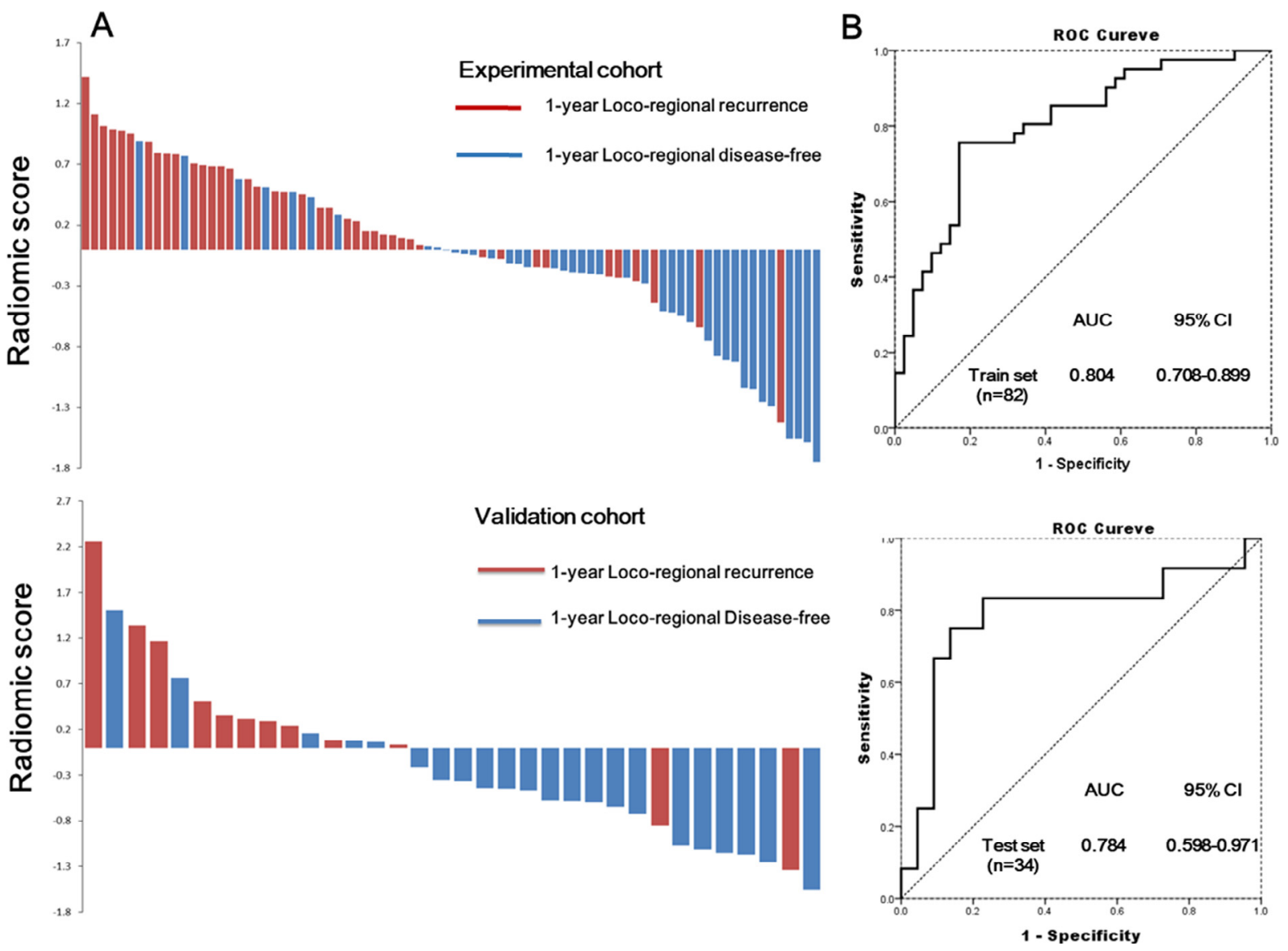

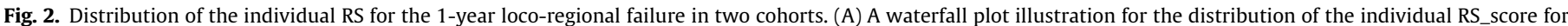

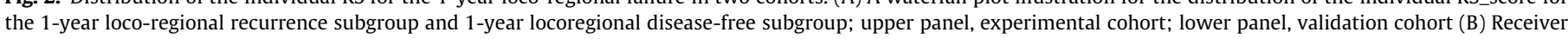

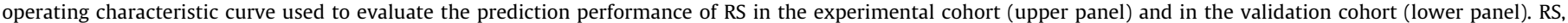
radiomic signature.

bootstrap-corrected C-index values of 0.7609 and 0.7545 in the experimental and validation cohorts, respectively.

We further used the Youden index according to the ROC curve of the RS in the experimental cohort to obtain an optimal cutpoint by maximizing the sum of the sensitivity and specificity. The overall accuracies by using an RS cutoff point (RS_cutoff) of 0.0326 to predict the 1 year LRF in experimental, validation, stage III/IV, and cT4a cohort were $79.27 \%, 79.41 \%, 76.74 \%$, and $71.15 \%$, respectively (Table 3 ). The 1 -year LRF prediction performance of the RS_cutoff outperformed the traditional volumetric predictors by using a tumor volume of $25 \mathrm{~cm}^{3}, 30 \mathrm{~cm}^{3}$, and $35 \mathrm{~cm}^{3}$ in the experimental, validation, stage III/IV, and cT4a cohort, and detailed information are listed in Table 3.
3.4. Progression-free, laryngectomy-free, and overall survival in risk groups categorized via the RS_cutoff

We used a dichotomized risk category, with an RS_cutoff of 0.0326 , to categorize patients into low- and high-risk groups. In addition to the prediction of 1- and 2-year LRF, we found that the high-risk group was significantly associated with worse survival outcome, including 1- and 2-year PFS, LFS, and OS in both institution cohorts (Fig. 3).

In patients with stage III/IVA disease, the median PFS $(P<.001)$, LFS $(P=.001)$, and OS $(P<.001)$ were $57.0,65.8$, and 68.4 months in the low-risk group compared to $18.4,27.2$, and 30.7 months in the high-risk group, respectively (Supplementary Fig. 1). In

Table 3

1-year Loco-regional failure prediction performance of radiomic signature and volumetric predictors.

\begin{tabular}{|c|c|c|c|c|c|c|c|c|c|c|}
\hline & \multicolumn{5}{|c|}{ Experimental cohort } & \multicolumn{5}{|c|}{ Validation cohort } \\
\hline & Sensitivity & Specificity & PPR & NPR & Accuracy & Sensitivity & Specificity & PPR & NPR & Accuracy \\
\hline RS_cutoff & $75.60 \%$ & $82.90 \%$ & $81.60 \%$ & $77.30 \%$ & $79.27 \%$ & $83.33 \%$ & $77.30 \%$ & $66.70 \%$ & $89.50 \%$ & $79.41 \%$ \\
\hline V35 & $53.70 \%$ & $80.50 \%$ & $73.33 \%$ & $63.50 \%$ & $67.07 \%$ & $58.30 \%$ & $81.80 \%$ & $63.60 \%$ & $78.30 \%$ & $73.53 \%$ \\
\hline V30 & $58.50 \%$ & $70.70 \%$ & $66.70 \%$ & $63.00 \%$ & $64.63 \%$ & $66.70 \%$ & $81.80 \%$ & $66.70 \%$ & $81.80 \%$ & $76.47 \%$ \\
\hline \multirow[t]{2}{*}{ V25 } & $73.20 \%$ & $56.10 \%$ & $\begin{array}{l}62.50 \% \\
\text { Stage III/IVA }\end{array}$ & $67.60 \%$ & $64.63 \%$ & $75.00 \%$ & $77.30 \%$ & $\begin{array}{l}64.30 \% \\
\text { cT4a }\end{array}$ & $85.00 \%$ & $76.47 \%$ \\
\hline & Sensitivity & Specificity & PPR & NPR & Accuracy & Sensitivity & Specificity & PPR & NPR & Accuracy \\
\hline RS_cutoff & $70.00 \%$ & $82.60 \%$ & $77.80 \%$ & $76.00 \%$ & $76.74 \%$ & $75.90 \%$ & $65.20 \%$ & $73.30 \%$ & $68.20 \%$ & $71.15 \%$ \\
\hline V35 & $45.00 \%$ & $82.60 \%$ & $69.20 \%$ & $63.30 \%$ & $65.12 \%$ & $58.60 \%$ & $65.00 \%$ & $68.00 \%$ & $55.60 \%$ & $61.54 \%$ \\
\hline V30 & $50.00 \%$ & $73.90 \%$ & $63.00 \%$ & $62.50 \%$ & $62.79 \%$ & $62.10 \%$ & $47.80 \%$ & $60.00 \%$ & $50.00 \%$ & $55.77 \%$ \\
\hline V25 & $65.00 \%$ & $60.00 \%$ & $59.10 \%$ & $66.70 \%$ & $62.79 \%$ & $75.90 \%$ & $26.10 \%$ & $56.40 \%$ & $46.20 \%$ & $51.92 \%$ \\
\hline
\end{tabular}

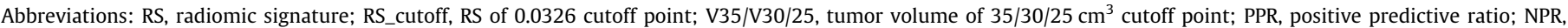
negative predictive ratio. 

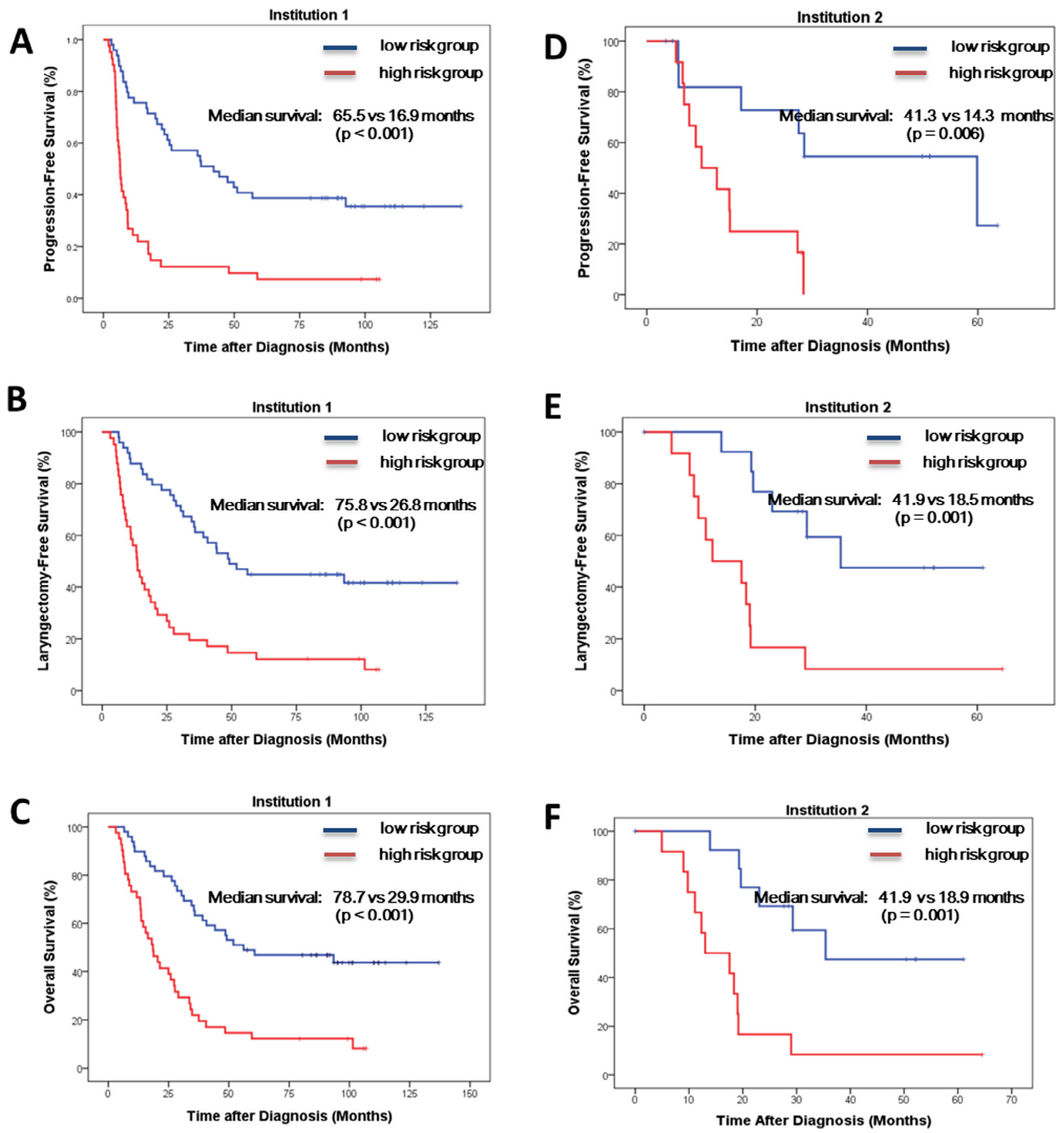

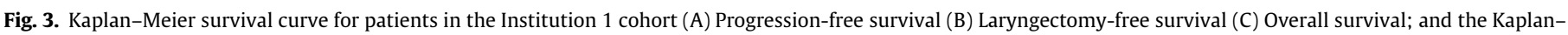
Meier survival curve for patients in the Institution 2 cohort (D) Progression-free survival (E) Laryngectomy-free survival (F) Overall survival.

patients diagnosed with a cT4a disease, the median PFS $(P=.013)$, LFS $(P=.01)$, and OS $(P=.015)$ were 52.4, 62.6, and 65.0 months in the low-risk group compared to 20.0, 30.1, and 34.0 months in the high-risk group, respectively (Supplementary Fig. 1).

\section{Discussion}

Radiomics is a quantitative analytical method used to convert medical images into a large-scale profile of imaging features [15]. In the current study, we demonstrated that RS, a combination of 8 potential factors obtained from 800 image features, showed good discrimination performance for the prediction of the 1-year LRF not only in the experimental cohort, but also in the validation cohort. By using the Youden index, we categorized patients into a high-risk and a low-risk category based on an RS_cutoff of
0.0326. Importantly, the high-risk group was closely associated with a poor median PFS, LFS and OS in two independent institution cohort, stage III/IV subgroup, and cT4a subgroup. To the best of our knowledge, the present study is the first of its kind, reporting an MRI-derived RS-based prediction model for the evaluation of the 1 -year LRC and survival in patients with HPSCC who received OPT as a first-line treatment.

Previous studies have revealed that a larger tumor size is closely associated with poor disease outcomes in patients with HNC who received OPT [13,14,29,30,31]. Strongin et al. reported that among 78 patients with stage III/IV HNC who received OPT, patients with a TV $>35 \mathrm{~cm}^{3}$ were associated with higher recurrent rates compared to those with a TV $\leq 35 \mathrm{~cm}^{3}$ (43\% vs. $71 \%, P=.010$ ) [13]. Johnson et al. also studied the association between tumor volume and disease recurrence in 76 HNC patients, and found that the 5-year DFS was significantly better for volumes of $<35 \mathrm{~cm}^{3}(P=.0001)$ [31]. 
Chen et al. reported that among the III-IVA HPSCC patients treated with CCRT, a TV $<30 \mathrm{~cm}^{3}$ significantly correlated with a better 3year PFS than a TV $\geq 30 \mathrm{~cm}^{3}$ [14]. In addition to TV, Zhang et al. revealed that histogram analysis of parameters of primary tumor CT textures, including entropy and skewness, were associated with a better OS in HNC patients receiving IC [32].

In the present study, we found that among patients with stage III/IVA and CT4 disease, the prediction accuracy of the 1-year LRF were $65.12 \%$ and $61.54 \%$ by using TV $<$ or $\geq 35 \mathrm{~cm}^{3}$ and $62.79 \%$ and $55.77 \%$ by using $\mathrm{TV}<$ or $\geq 30 \mathrm{~cm}^{3}$, respectively. The results were compatible with the historical prognosis predictive performance of tumor volume. Nevertheless, our study demonstrated that RS (RS_cutoff of 0.0326) provided a better prediction accuracy for the 1-year LRF in the stage III/IV subgroup (76.74\%), and the cT4a subgroup (71.15\%) than TV. The possible reason for these findings could be that texture analysis for tumor imaging could provide precise information on intra-tumor heterogeneity, which may affect the response of tumors to chemotherapy and RT [27,33-36]. For example, Aerts et al. identified a close correlation between the intra-tumor textural features and the expression patterns of different genes related to tumor growth and invasiveness in patients with HNC [20], which were thought to be important prognostic factors regarding post treatment disease control.

In addition to the radiomic approach via anatomical radiographic imaging, a lower pretreatment metabolic TV (MTV) value $[37,38]$, a volumetric measurement of tumor extent with ${ }^{18}$ F-FDG uptake scans, as well as a lower maximum standardized uptake value (SUV) was found to be closely associated with complete or partial responses in patients with HPSCC who received IC followed by definitive CCRT [32]. An early response to IC is another approach for the selection of patients suitable for OPT. Recently, Wichmann et al. developed a predictive model, the LFS score, based on the response to a cycle of IC using a combination of $>2 \mathrm{LN}+$, residual primary TV $>20 \%$, residual total TV $>5.6 \mathrm{~mL}$, and the ratio of the maximal residual standard-uptake value maximum to the mean residual SUV mean $>1.51$, and showed that a LFS score $\leq 16$ significantly correlated with better LFS $(P=.0014)$ and OS $(P=.0146)$ [39]. Combining radiomic analysis and functional MRI modalities such as diffusion-weighted imaging (DWI) would have the potential effect of providing more information about the prognostic imaging phenotype in patients with advanced HPSCC. Recently, Leithner et al reported that radiomic analysis derived from DWI imaging can provide high accuracy in subclassifying the molecular subtypes of breast cancer (luminals A and B, and HER2 enriched) [40]. Dulhanty et al also demonstrated that radiomics-driven DWI can be used as a forcefully sensing strategy to help physicians precisely screen zone-level prostate cancers for further targeted biopsy treatment [41]. Further study is warranted to assess whether DWI-driven radiomic signatures can predict 1-year LRF in patients with advanced HPSCC who want to receive OPT. Taken together, these findings suggest that a combination of image features through the analysis of CT images, MRI images (included DWI), and ${ }^{18} \mathrm{~F}$-FDG PET might provide enormous information about the tumor phenotypic features [40-44], and the aforementioned approach should be confirmed in future studies for patients with HPSCC who received CCRT for organ preservation.

The present study consisted of two independent cohorts of patients with HPSCC receiving OPT, with a curative radiation dose $\geq 70$ Gy for the primary tumors. Nevertheless, there were some differences in the MRI imaging platform and sequence parameters between these two cohorts. To overcome the common problems frequently noticed in multi-institutional studies, we rescaled the image intensity data into $256 \mathrm{~Gy}$ levels and used a LoG image filter to improve image noise suppression in the current study. In the present study, our RS model, which comprises radiomic features derived only from primary tumors, can predict the prognosis of patients with
HPSCC who received a cisplatin-based CCRT as the first-line treatment, for the preservation of hypopharyngeal organ function. It would not be surprising that the addition of clinicopathological features such as tumor invasion status or lymph node disseminated the extent and radiographic information from regional lymphadenopathies will improve the current RS model.

Previous studies demonstrated that male sex, advanced age, tumor size, tumor invasion, lymph node stage, comorbid conditions, poor performance scores, human papillomavirus infection, and programmed death ligand-1 expression level are poor prognostic factors in patients with HPSCC [45-47]. Although radiomic research studies are still emerging, our RS model provided higher sensitivity and specificity than tumor volume in predicting locoregional recurrence in patients with advanced HPSCC, indicating that, in addition to clinicopathological factors, RS is a useful tool for predicting local failure in patients with advanced HPSCC who want to receive OPT.

Previous studies also demonstrated that multiple factors including laryngeal function, cartilage invasion (T4a), tumor volume, lymph node involvement, and distant metastases are associated with the prognosis and feasibility of organ preservation therapy $[48,49]$. When adding the variables of clinical T (T4 versus T1-3) and clinical N (N1-3 versus N0) in our RS in the regression model, we found that the tumor and lymph node status with RS combined yielded c-index values of 0.803 and 0.782 in the experimental and validation cohorts, respectively, indicating that the tumor and lymph node status combined and the RS model did not improve the discriminative performance of the combined model in predicting LRF when compared with our original RS model. The reasons for the aforementioned results may be related to the limited size of our current cohorts, in which prognostic factors such as tumor invasion and lymph node extension may have rarely existed in our patients with advanced HPSCC. However, with adequate sizes of the experimental and validation cohorts, the addition of TNM stage (including tumor size, tumor invasion, and lymph node stage) and radiographic information from regional lymphadenopathies will potentially improve our established RS model. Further large-scale external validation and advanced radiomic analyses are warranted to develop a clinico-radiomic model for increasing the accuracy and robustness of the prediction of loco-regional recurrence and prognosis in patients with HPSCC.

In the present study, MRI scans were obtained at the time of initial diagnosis of all patients, and none of the patients who underwent tracheotomy were included in our cohort. Considering that problems of image artifacts from the endotracheal tube in patients who had undergone tracheostomy may interfere with the data presentation of radiomic analyses, in addition to bias field correction and imaging gray-level normalization, we will contour the endotracheal tube with a 5-mm margin and subtract the endotracheal tube region from the tumor extent for further radiomic analysis in this subgroup of patients.

In summary, our established and validated non-invasive RS model provides a novel and convenient approach to predict 1year LRF, and the survival outcome, including the PFS, LFS, and OS, in patients with locally advanced HPSCC who received OPT. Validation of the association of our established RS (RS_cutoff $=0.0326$ ) with clinical outcomes in another large cohort of patients with HPSCC who received OPT is warranted. This established non-invasive RS can help physicians characterize and facilitate decision-making for the clinical management of patients with locally advanced HPSCC in future prospective studies. In the future, the use of an innovative radiomic analysis combined with primary tumor characteristics and regional lymphadenopathies in the assessment of the predictive ability for LRF in patients with advanced HPSCC and in the validation of the robustness of this combined RS model is warranted. 


\section{Declaration of Competing Interest}

The authors declare that they have no known competing financial interests or personal relationships that could have appeared to influence the work reported in this paper.

\section{Funding sources}

This study was supported by the following research grants MOST 107-2314-B-002-217-MY3 from the Ministry of Science and Technology, Taiwan, NTUH 109-S4716 from National Taiwan University Hospital, Taiwan, and NTUHYL 106.S006 from the National Taiwan University Hospital Yun-Lin Branch.

\section{Authors' contributions}

C.-Y.H, and S.-M.L. performed the study concept and design. C.Y.H., S.-M.L., and S.-H.K. were responsible for data acquisition, data analysis, and interpretation. C.-Y.H., S.-M.L., W.-C.W., and S.-H.K. contributed to the bioinformatic analysis. C.-Y.H., S.-M.L., N.M.T., Y.-H.J., C.-W.W., W.-C.W., and S.-H.K. were responsible for manuscript writing and approval of final version of submitted manuscript. S.-H.K. designed, planned, and coordinated the study over the entire period.

\section{Appendix A. Supplementary data}

Supplementary data to this article can be found online at https://doi.org/10.1016/j.ctro.2020.08.004.

\section{References}

[1] Jemal A, Bray F, Center MM, Ferlay J, Ward E, Forman D. Global cancer statistics. CA Cancer J Clin 2011;61:69-90.

[2] Lefebvre JL, Andry G, Chevalier D, et al. Laryngeal preservation with induction chemotherapy for hypopharyngeal squamous cell carcinoma: 10-year results of EORTC trial 24891. Ann Oncol 2012;23:2708-14.

[3] Carvalho AL, Nishimoto IN, Califano JA, Kowalski LP. Trends in incidence and prognosis for head and neck cancer in the United States: a site-specific analysis of the SEER database. Int J Cancer 2005;114:806-16.

[4] Lee NY, O'Meara W, Chan K, et al. Concurrent chemotherapy and intensitymodulated radiotherapy for locoregionally advanced laryngeal and hypopharyngeal cancers. Int J Radiat Oncol Biol Phys 2007;69:459-68.

[5] Katsoulakis E, Riaz N, Hu M, et al. Hypopharyngeal squamous cell carcinoma: three-dimensional or Intensity-modulated radiotherapy? A single institution's experience. Laryngoscope 2016;126:620-6.

[6] Lefebvre J, Ang KK. Larynx preservation clinical trial design: key issues and recommendations-a consensus panel summary. Head Neck 2009;31:429-41.

[7] Weber RS, Berkey BA, Forastiere A, Cooper J, Maor M, Goepfert H, et al. Outcome of salvage total laryngectomy following organ preservation therapy: the Radiation Therapy Oncology Group trial 91-11. Arch Otolaryngol Head Neck Surg 2003;129:44-9.

[8] Withrow KP, Rosenthal EL, Gourin CG, et al. Free tissue transfer to manage salvage laryngectomy defects after organ preservation failure. Laryngoscope 2007; $117: 781-4$.

[9] Mok G, Gauthier I, Jiang H, et al. Outcomes of intensity-modulated radiotherapy versus conventional radiotherapy for hypopharyngeal cancer. Head Neck 2015;37:655-61.

[10] Boehm A, Lindner F, Wichmann G, et al. Impact of indication-shift of primary and adjuvant chemo radiation in advanced laryngeal and hypopharyngeal squamous cell carcinoma. Eur Arch Otorhinolaryngol 2015;272:2017-25.

[11] Johnson CR, Thames HD, Huang DT, Schmidt-Ullrich RK. The tumor volume and clonogen number relationship: tumor control predictions based upon tumor volume estimates derived from computed tomography. Int J Radiat Oncol Biol Phys 1995;33:281-7.

[12] Studer G, Lutolf UM, El-Bassiouni M, Rousson V, Glanzmann C. Volumetric staging (VS) is superior to TNM and AJCC staging in predicting outcome of head and neck cancer treated with IMRT. Acta Oncol 2007;46:386-94.

[13] Strongin A, Yovino S, Taylor R, et al. Primary tumor volume is an important predictor of clinical outcomes among patients with locally advanced squamous cell cancer of the head and neck treated with definitive chemoradiotherapy. Int J Radiat Oncol Biol Phys 2012;82:1823-30.

[14] Chen S, Yang S, Liang J, Lin F, Tsai M. Prognostic impact of tumor volume in patients with stage III-IVA hypopharyngeal cancer without bulky lymph nodes treated with definitive concurrent chemoradiotherapy. Head Neck 2009;31:709-16.
[15] Lambin P, Rios-Velazquez E, Leijenaar R, et al. Radiomics: extracting more information from medical images using advanced feature analysis. Eur J Cancer 2012;48:441-6.

[16] Kumar V, Gu Y, Basu S, et al. Radiomics: the process and the challenges. Magn Reson Imag 2012;30:1234-48.

[17] Parmar C, Leijenaar RT, Grossmann P, et al. Radiomic feature clusters and prognostic signatures specific for Lung and Head \& Neck cancer. Sci Rep 2015;5:11044.

[18] Kickingereder P, Gotz M, Muschelli J, et al. Large-scale radiomic profiling of recurrent glioblastoma identifies an imaging predictor for stratifying antiangiogenic treatment response. Clin Cancer Res 2016;22:5765-71.

[19] Huang YQ, Liang CH, He L, et al. Development and validation of a radiomics nomogram for preoperative prediction of lymph node metastasis in colorectal cancer. J Clin Oncol 2016;34:2157-64.

[20] Aerts HJ, Velazquez ER, Leijenaar RT, et al. Decoding tumour phenotype by noninvasive imaging using a quantitative radiomics approach. Nat Commun 2014;5:4006.

[21] Fedorov A, Beichel R, Kalpathy-Cramer J, et al. 3D Slicer as an image computing platform for the Quantitative Imaging Network. Magn Reson Imag 2012;30:1323-41.

[22] Lee J, Jain R, Khalil K, et al. Texture feature ratios from relative CBV maps of perfusion MRI are associated with patient survival in glioblastoma. AJNR Am J Neuroradiol 2016;37:37-43.

[23] Talwalkar JA, Yin M, Fidler JL, Sanderson SO, Kamath PS, Ehman RL. Magnetic resonance imaging of hepatic fibrosis: emerging clinical applications. Hepatology 2008;47:332-42.

[24] Srinivasan G, Shobha G. Statistical texture analysis. vol. 36, 2008, p. 1264-9

[25] Aggarwal N, Agrawal R. First and second order statistics features for classification of magnetic resonance brain images, 2012.

[26] Tang X. Texture information in run-length matrices. IEEE Trans Image Process 1998; 7:1602-9.

[27] Breiman L. Better subset regression using the nonnegative garrote. Technometrics 1995;37:373-84.

[28] Harrell F. Regression modeling strategies: with applications to linear models, logistic and ordinal regression, and survival analysis. Springer; 2015.

[29] Plataniotis GA, Theofanopoulou ME, Kalogera-Fountzila A, Haritanti A, Ciuleanou E, Ghilezan N, et al. Prognostic impact of tumor volumetry in patients with locally advanced head-and-neck carcinoma (nonnasopharyngeal) treated by radiotherapy alone or combined radiochemotherapy in a randomized trial. Int J Radiat Oncol Biol Phys 2004;59:1018-26.

[30] Grabenbauer GG, Steininger H, Meyer M, et al. Nodal CT density and total tumor volume as prognostic factors after radiation therapy of stage III/IV head and neck cancer. Radiother Oncol 1998;47:175-83.

[31] Johnson CR, Khandelwal SR, Schmidt-Ullrich RK, Ravalese 3rd J, Wazer DE. The influence of quantitative tumor volume measurements on local control in advanced head and neck cancer using concomitant boost accelerated superfractionated irradiation. Int J Radiat Oncol Biol Phys 1995;32:635-41.

[32] Zhang H, Graham CM, Elci O, et al. Locally advanced squamous cell carcinoma of the head and neck: CT texture and histogram analysis allow independent prediction of overall survival in patients treated with induction chemotherapy. Radiology 2013;269:801-9.

[33] Hockel M, Knoop C, Schlenger K, Vorndran B, Baussmann E, Mitze M, et al. Intratumoral pO2 predicts survival in advanced cancer of the uterine cervix. Radiother Oncol 1993;26:45-50.

[34] Nie K, Shi L, Chen Q et al. Rectal cancer: assessment of neoadjuvant chemoradiation outcome based on radiomics of multiparametric MRI. Clin Cancer Res 2016;22:5256-64.

[35] Press RH, Shu H-K-G, Shim H, et al. The use of quantitative imaging in radiation oncology: a quantitative imaging network (QIN) perspective. Int J Radiat Oncol Biol Phys 2018;102:1219-35.

[36] Nie K, Al-Hallaq H, Li XA, et al. NCTN assessment on current applications of Radiomics in oncology. Int J Radiat Oncol Biol Phys 2019;104:302-15.

[37] Park GC, Kim JS, Roh JL, Choi SH, Nam SY, Kim SY. Prognostic value of metabolic tumor volume measured by 18F-FDG PET/CT in advanced-stage squamous cell carcinoma of the larynx and hypopharynx. Ann Oncol 2013;24:208-14.

[38] Chung MK, Jeong HS, Park SG, et al. Metabolic tumor volume of [18F]fluorodeoxyglucose positron emission tomography/computed tomography predicts short-term outcome to radiotherapy with or without chemotherapy in pharyngeal cancer. Clin Cancer Res 2009;15:5861-8.

[39] Wichmann G, Kruger A, Boehm A, et al. Induction chemotherapy followed by radiotherapy for larynx preservation in advanced laryngeal and hypopharyngeal cancer: outcome prediction after one cycle induction chemotherapy by a score based on clinical evaluation, computed tomography-based volumetry and 18F-FDG-PET/CT. Eur J Cancer 2017;72:144-55.

[40] Leithner D, Bernard-Davila B, Martinez DF, et al. Radiomic signatures derived from Diffusion-Weighted Imaging for the assessment of breast cancer receptor status and molecular subtypes. Mol Imag Biol 2020;22:453-61.

[41] Dulhanty C, Wang L, Cheng M, et al. Radiomics driven diffusion weighted imaging sensing strategies for zone-level prostate cancer sensing. Sensors (Basel) 2020;20.

[42] Leger S, Zwanenburg A, Pilz K, et al. CT imaging during treatment improves radiomic models for patients with locally advanced head and neck cancer. Radiother Oncol 2019;130:10-7. 
[43] Bogowicz M, Leijenaar RTH, Tanadini-Lang S, et al. Post-radiochemotherapy PET radiomics in head and neck cancer-the influence of radiomics implementation on the reproducibility of local control tumor models. Radiother Oncol 2017;125:385-91.

[44] Krarup MMK, Nygård L, Vogelius IR, et al. Heterogeneity in tumours: validating the use of radiomic features on 18F-FDG PET/CT scans of lung cancer patients as a prognostic tool. Radiother Oncol 2019;144:72-8.

[45] Abdeyrim A, He S, Zhang Y, et al. Prognostic value of lymph node ratio in laryngeal and hypopharyngeal squamous cell carcinoma: a systematic review and meta-analysis. J Otolaryngol Head Neck Surg 2020;49:31.
[46] Hughes RT, Beuerlein WJ, O’Neill SS, et al. Human papillomavirus-associated squamous cell carcinoma of the larynx or hypopharynx: clinical outcomes and implications for laryngeal preservation. Oral Oncol 2019;98:20-7.

[47] Ono T, Azuma K, Kawahara A, et al. Association between PD-L1 expression combined with tumor-infiltrating lymphocytes and the prognosis of patients with advanced hypopharyngeal squamous cell carcinoma. Oncotarget 2017;8:92699-714.

[48] de Bree R. The current indications for non-surgical treatment of hypopharyngeal cancer. Adv Otorhinolaryngol 2019;83:76-89.

[49] Kang S. Organ preservation in laryngeal and hypopharyngeal cancer. Oral Oncol 2019;90:6-7. 